\title{
PERSPECTIVE
}

\section{The Spectrum of Harm Associated with Modern Medicine}

\author{
Ami Schattner, MD
}

The Faculty of Medicine, Hebrew University-Hadassah Medical School, Jerusalem, Israel.

J Gen Intern Med 37(3):664-7

DOI: $10.1007 /$ s11606-021-06997-x

(C) Society of General Internal Medicine 2021

$\mathrm{C}$ omparing a textbook of internal medicine published in 1970 to one appearing in 2020 can be a fascinating experience. The achievements of modern medicine and clinical science over the last 50 years are widely acknowledged. Consider the progress in imaging and interventional capabilities in most disciplines; the major advances in understanding the molecular and immunological pathogenesis of disease, translated into new tests and sophisticated medications; the adoption of evidence-based evaluation of tests and treatments; and the myriad advantages of incorporating communication and information technologies. However, side by side with the mounting changes, a substantial body of harm has developed in recent years, associated with exactly the same valuable advances. Termed "Prosperity and despair" or "the chaos and dysfunction that characterize today's medical care,"1 the multifaceted harm associated with modern medicine has seldom been discussed as a whole, although some aspects (e.g., burnout) have been separately studied. We will briefly review the wide spectrum of harm that appeared and developed in association with the advances of medicine, detracting considerably from its many benefits, and suggest main themes of potential remedies.

Modern medicine-associated harm is often discussed in the context of its effects on health-care systems - such as escalating costs, which do not necessarily translate into better health outcomes, or drug and device corporations who may use aggressive marketing strategies to influence physicians and the public towards overuse of costly modalities. However, we will focus on the profound effects on patients and physicians, since marked changes, often deterioration, in the experience of caregiving as perceived by both patients and physicians have occurred, deeply affecting the traditional clinical encounter. ${ }^{2}$ These can be classified into five domains of change, so that the current harm associated with each can be addressed (Tables 1 , 2, 3, 4, and 5).

Received April 19, 2021

Accepted June 22, 2021

Published online August 2, 2021
The inherent strain between constant time constraints and increasing demands and complexity facing physicians is the change most profoundly affecting encounters. Defined as inability to complete necessary work in the time allotted, it was identified in just 113 publications over 1970-1990 vs. 2570 over the last decade. Time constraints and pressures, reported worldwide for multiple settings, are both absolute and relative. While hospital length of stay has decreased and ambulatory consultation times remained essentially unchanged, physicians' tasks have markedly escalated. Ageing, multimorbidity, and polypharmacy have added to each patient's complexity and, hence, time requirements. Current physicians' obligations multiplied to include providing essential patient-centered care that must comprise supporting the patient's health literacy, shared decision-making, and taking contextual factors into account-all time consuming. ${ }^{3}$ The steadily increasing, constantly changing scientific and clinical knowledge base and options to choose from according to the latest evidence also contribute to physicians' burden. Hospital encounters do no better than encounters in ambulatory settings: time-motion studies indicate that computer time and administrative chores now occupy $50 \%$ of internal medicine residents' time, with frequent multitasking, while patient face time deteriorated to $<10 \%,{ }^{4}$ and with it clinical skills, physician's confidence, humanistic aspects of the encounter, and patient (and physician) satisfaction. Thus, critical elements of patients' trust, adherence, health outcomes, and the quality of care (QOC) have been affected (Table 1).

One unfortunate result may be that physicians' burnout (3250 citations 2010-2020) has become widespread, with USA prevalence estimates of over $50 \%$ on overall burnout or depersonalization and $72 \%$ on emotional exhaustion. The consequences are severe not only personally for physicians but to patients who suffer inevitable detrimental effects in every component of the encounter starting with the quality of the history and examination, through expression of empathy, provision of preventive services, possibly unwarranted tests and referrals, suboptimal treatment selection, and increased errors and adverse events. ${ }^{5}$ Thus, the QOC, and patients' satisfaction, health, and coping are often significantly affected.

With increasing complexity and uncertainty, dwindling time, and impending burnout, ordering tests (that often beget more tests) or adding more medications, and referrals, may often provide a welcome diversion ("shunt") to health providers, gaining valuable time and sharing responsibility. 
Table 1 Five domains of change in the current practice of medicine, and their potential adverse consequences**. First domain: time constraints

\section{Short visits-rushed encounter}

$\downarrow$ Length of stay (hospital) or unchanged encounter time (ambulatory care) vs. more issues per visit:

$\uparrow$ New, time-consuming tasks (e.g., patient-centered care; preventive services); $\uparrow$ Patients' complexity (ageing, multimorbidity, polypharmacy); flooding of patient-related data (e.g., in the EHR); proliferating EHR prompts demanding attention;

$\uparrow$ Options for tests and treatments beget harder decisions (see Table 4);

$\downarrow$ Face time with patients, especially vs. $\uparrow$ screen time, (see Table 3);

Communication;

$\downarrow$ Patient-physician relationship/bonding;

Attention to patients' cues and concerns, or to nonverbal clues;

$\downarrow$ Empathy;

$\downarrow$ Communication skills; further $\downarrow$ In communication (vicious cycle);

$\downarrow$ Patient-centered care;

$\downarrow$ Time for physical examination;

$\downarrow$ Clinical skills;

$\downarrow$ Self-assurance and confidence; further $\downarrow$ In examination (vicious cycle);

Satisfaction;

$\downarrow$ Time for consulting databases or colleagues;

$\uparrow$ Dependence on assistants (e.g., scribes, clinical pharmacists)

\section{Physician burnout}

$\uparrow$ Demands for productivity- $-\uparrow$ workloads;

$\uparrow$ Daily stress;

$\downarrow$ Control over time and substantial decline in autonomy;

$\uparrow$ Managerial scrutiny and supervision (cost, "defending" choices,

quality metrics, patient satisfaction);

$\uparrow$ Role of evidence-based medicine: downplaying individual experience and clinical judgment;

$\uparrow$ Clerical and administrative duties;

$\uparrow$ Remuneration concerns;

$\uparrow$ Screen time vs. $\downarrow$ face time;

$\uparrow$ Patient's demands and criticism (see Table 5);

$\uparrow$ Fatigue, exhaustion;

$\uparrow$ Irritability, anger, conflicts;

Teamwork capacity;

$\downarrow$ Efficiency and productivity;

$\uparrow$ Incidence of medical errors;

$\downarrow$ Empathy, compassion, and humanism;

$\downarrow$ Patient-physician relationship/bonding;

$\downarrow$ Attention to patients' cues and concerns;

$\downarrow$ Sense of altruistic achievement;

$\uparrow$ Guilt;

Meaning;

$\uparrow$ Distancing;

Satisfaction;

$\downarrow$ Coping;

$\uparrow$ Attrition;

$\uparrow$ Intent to leave and job turnover;

$\uparrow$ Early retirement;

$\downarrow$ Attention/time for personal life, personal health;

Personal health ("self-care");

$\uparrow$ Marital/relations problems;

$\uparrow$ Alcohol, smoking, substance abuse;

$\uparrow$ Depression;

$\uparrow$ Risk of accidents (driving, needle sticks), suicidal ideation, mortality ratios;

$\uparrow$ Unprofessional behaviors

-All creating a vicious cycle

III. Impaired clinical performance and quality of care (largely due to I. + II.)

$\downarrow$ /Impaired decision-making;

$\downarrow$ Adherence to clinical guidelines;

$\downarrow$ Patient-centered care;

$\uparrow$ Referrals of all kinds, including unnecessary imaging, tests, consultations, and procedures (see Table 2);

$\uparrow$ Prescriptions, some unnecessary;

$\uparrow$ Risk of adverse effects and iatrogenic harm;

$\uparrow$ "Defensive" practice;

$\downarrow$ Energy for consultation (databases, colleagues) to select appropriate test/treatment;

$\uparrow$ Use of heuristics ("type 1, intuitive reasoning") and bias;

$\uparrow$ Diagnostic and other errors, and adverse events;

\section{Table 1. (continued)}

$\downarrow$ Recommendation of evidence-based screening or healthy lifestyle;

$\uparrow$ Readmissions;

$\downarrow$ Patient safety;

$\downarrow$ Patient satisfaction and adherence

**Reported for every discipline (primarily, family medicine and general internal medicine) and setting, including hospital admissions. Domains effects are interwoven with each other. All contribute together to the "crisis," "collapse," or "chaos" (quotes) in current health-care systems

However, cost, false-positive incidental findings, increased risk of cascades of adverse events, and mounting patient anxiety are just a few of the deleterious outcomes. The computer, electronic health record (EHR), and rapid availability of information and communication are doubtless powerful tools. But at the same time, being engrossed in the screen instead of the patient; having to comply with countless computergenerated demands; or being flooded with hundreds of information items that have to be quickly assimilated, present hitherto-unseen difficulties for physicians. ${ }^{6}$ Patients have also changed, being often more multicultural, knowledgeable, demanding, and certainly, more on par with their physician in terms of access to information and shared decisions instead of the prior paternalistic attitude. This evolution of patients' roles is generally desirable, but unavoidably adds to physicians' burden, having to provide more detailed explanations to more critical and better-informed patients. Economic inequality in the USA and elsewhere has also been increasing, contributing to significant health-care disparities or inequities - an inherent harm, difficult for both sides. All these changes (Tables 1, 2, 3, 4 , and 5) add to the strain and potential harm of current clinical encounters, and are essentially common to all settings and disciplines.

Table 2 Five domains of change in the current practice of medicine, and their potential adverse consequences**. Second domain: escalating tests, drugs, and interventions

$\uparrow$ Dependence on tests;

$\downarrow$ Clinical skills: declining attention to the patient's narrative and examination;

$\downarrow$ Self-confidence;

$\uparrow$ Distancing;

$\downarrow$ Satisfaction;

$\uparrow$ Unnecessary tests and redundant procedures;

$\uparrow$ Incidental ("false-positive") findings (begetting more tests: a vicious cycle);

$\uparrow$ Cascades of care and potential of iatrogenic harm;

$\uparrow$ Costs to the health-care system and patients;

$\uparrow$ Disparities in health care;

$\uparrow$ Prescriptions/polypharmacy, often favoring new and expensive medications;

$\uparrow$ Congestion and delays compromising access;

$\uparrow$ Interventions not significantly prolonging life or allowing quality of life ("futility");

$\uparrow$ Options beget harder decisions

**Reported for every discipline (primarily, family medicine and general internal medicine) and setting, including hospital admissions. Domains' effects are interwoven with each other. All contribute together to the "crisis," "collapse," or "chaos" (quotes) in current health-care systems 
Table 3 Five domains of change in the current practice of medicine, and their potential adverse consequences**. Third domain: computers

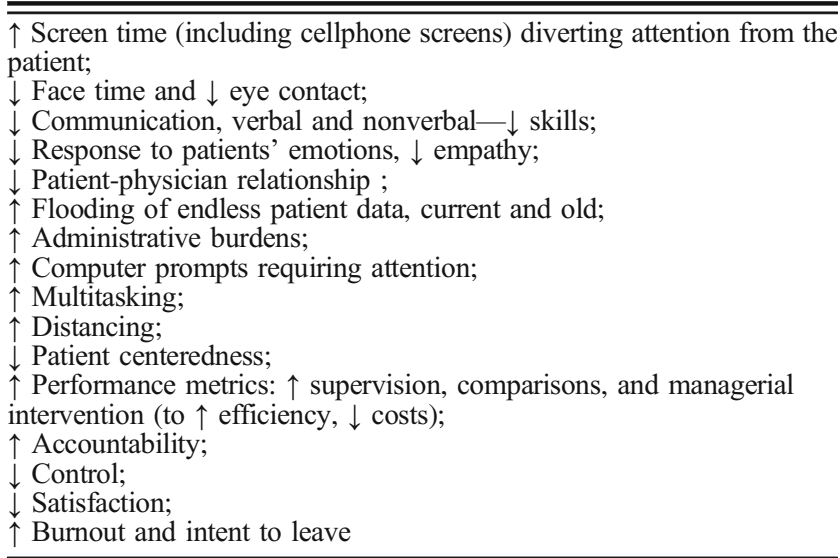

**Reported for every discipline (primarily, family medicine and general internal medicine) and setting, including hospital admissions. Domains' effects are interwoven with each other. All contribute together to the "crisis," "collapse," or "chaos" (quotes) in current health-care systems

It is much easier to identify deficiencies than to try and correct them, but clearly a multipronged approach in several domains is required to adapt to the deleterious changes brought by the rapid developments and achievements of modern medicine. It is simplistic to assume that remuneration will suffice, and there is little evidence to support that measures targeting providers' habits are effective. ${ }^{7}$ However, several concurrent approaches may be adopted. First, national and organizational interventions are required to reduce physicians' workload by acknowledging increased complexity and tasks and affording physicians longer time per patient, more flexibility, and more control over their time. ${ }^{3}$ This would be similar to the "new" duty-hours regulations for residents. Providing clerical support and other assistance to relieve some duties (e.g., scribes) seems another useful direction. Additional system interventions may develop a more efficient, user-friendly EHR, simplifying and minimizing documentation and insurers'-initiated boxes "de-

Table 4 Five domains of change in the current practice of medicine, and their potential adverse consequences**. Fourth domain: expanding knowledge

$\uparrow$ Time required to tailor diagnosis/treatment to the unique individual— $-\uparrow$ options for tests and treatments beget harder decisions ;

$\uparrow$ Biased inferences by drugs and devices industries: $\uparrow$ potential of

skewed recommendations; $\uparrow$ guidelines to follow (some contradictory);

$\downarrow$ Constancy-goals and methods keep changing: a "moving target";

$\uparrow$ Role of evidence-based medicine: downplaying individual experience and clinical judgment;

$\uparrow$ Specialization and mandatory sub-sub-specialties; $\uparrow$ fragmentation of care;

$\downarrow$ Impaired transitions of care ("handoffs");

$\downarrow$ Control;

$\downarrow$ Accountability;

$\downarrow$ Confidence;

$\uparrow$ Risk of errors;

$\uparrow$ Readmissions

**Reported for every discipline (primarily, family medicine and general internal medicine) and setting, including hospital admissions. Domains' effects are interwoven with each other. All contribute together to the "crisis," "collapse," or "chaos" (quotes) in current health-care systems
Table 5 Five domains of change in the current practice of medicine, and their potential adverse consequences**. Fifth domain: changing patients

\begin{tabular}{|c|}
\hline $\begin{array}{l}\uparrow \text { Age, complexity, multimorbidity, polypharmacy and } \uparrow \text { cultural } \\
\text { diversity-harder communication, longer encounters, harder decisions; } \\
\uparrow \text { Economic inequality: } \uparrow \text { health-care inequities and discrepancies; } \\
\uparrow \text { Shared knowledge (e.g., through the internet); } \\
\uparrow \text { Shared authority (e.g., shared decision-making, required consent); } \\
\uparrow \text { Influence of aggressive, often biased marketing by health industry } \\
\text { corporations on patients; } \\
\uparrow \text { Patients' demands and perceived demands; } \\
\uparrow \text { Potential conflicts; } \\
\uparrow \text { Consumerism (e.g., changing physicians); } \\
\downarrow \text { Satisfaction; } \\
\downarrow \text { Trust; } \\
\downarrow \text { Adherence; } \\
\uparrow \text { Use of complementary and alternative medicine; } \\
\downarrow \text { Respect; } \\
\uparrow \text { Patients' complaints; } \\
\uparrow \text { Conflicts; } \\
\uparrow \text { Legislation }\end{array}$ \\
\hline
\end{tabular}

manding" to be ticked ${ }^{7}$; providing rapid access to updated, easy-to-use information-databases able to support decisions in real time (e.g., UpToDate); relaxing "big brother" surveillance via technology; and encouraging providers to take time for family, friends, sports, hobbies, and vacations and engaging in health-care-related creative arts. Otherwise, small-group organized physicians' meetings focusing on mindfulness, shared experiences, reflection, and teamwork (Balint-like) have shown promise in early trials and should be encouraged. Second, physicians' confidence in their clinical examination and communication skills must be enhanced, during both graduate and postgraduate education. The "Stanford Medicine 25," training in (art) observation, and fostering a prevailing attitude of curiosity towards problems and people may all rekindle the missing spice and meaning in daily work, revitalize physicians' inherent intrinsic motivation, and bring physicians and patients closer to each other. ${ }^{8}$ Third, new research is needed to develop and teach optimal techniques of using the computer without interfering with patient contact and humanistic communication (e.g., getting the previously available information before meeting the patient to ensure eye contact and focusing on the patient). Fourth, changes in medical education have to be implemented, including the principles and practice of narrative medicine and the art of listening, the power of empathy, judicious selection of tests and their interpretation in the clinical context, deprescribing, and a strong emphasis on teamwork.

In conclusion, the major advances in modern medicine came at a price, bringing with them a considerable body of harm, mostly related to the effects of increasing demands on health-care providers and their distancing from the patients. Appropriate system-related initiatives are needed, as well as changes in medical education, but physicians can already start by revitalizing their examination and communication skills, improving bonding with the patients, and attending to their own emotional needs. 
Corresponding Author: Ami Schattner, MD; The Faculty of Medicine, Hebrew University-Hadassah Medical School, Jerusalem, Israel (e-mail: amischatt@gmail.com).

Funding Not applicable

\section{Declarations:}

Conflict of Interest: The author declares that he does not have a conflict of interest.

\section{REFERENCES}

1. Larson EB, Fihn SD, Kirk LM, et al. The future of general internal medicine. Report and recommendations from the Society of General Internal Medicine (SGIM) Task Force on the domain of general internal medicine. J Gen Intern Med 2004; 19:69-77.
2. Schattner A. The clinical encounter revisited. Am J Med 2014; 127:26874 .

3. Konrad TR, Link CL, Shackelton RJ, et al. It's about time: physicians' perceptions of time constraints in primary care medical practice in three national healthcare systems. Med Care 2010; 48:95-100.

4. Mamykina L, Vawdrey DK, Hripcsak G. How do residents spend their shift time? A time and motion study with a particular focus on the use of computers. Acad Med 2016; 91:827-32.

5. Tsiga E, Panagopoulou E, Sevdalis $\mathrm{N}$, et al. The influence of time pressure on adherence to guidelines in primary care: an experimental study. BMJ Open 2013; 3:e002700.

6. Hartzband P, Groopman J. Off the record - avoiding the pitfalls of going electronic. N Engl J Med 2008: 358: 1656-8.

7. Panagioti M, Panagopoulou E, Bower P, et al. Controlled interventions to reduce burnout in physicians: a systematic review and meta-analysis. JAMA Intern Med 2017; 177: 195-205.

8. Schattner A. Curiosity. Are you curious enough to read on? J R Soc Med 2015; 108:160-4.

Publisher's Note: Springer Nature remains neutral with regard to jurisdictional claims in published maps and institutional affiliations. 